\title{
Three-Dimensional Architecture of the Tongue Muscles by Micro-CT with a Focus on the Longitudinal Muscle
}

\author{
Hidekazu Aoyagi ${ }^{*}$, Shin-ichi Iwasaki², Tomoichirou Asami ${ }^{3}$ \\ ${ }^{1}$ Advanced Research Center, School of Life Dentistry at Niigata, The Nippon Dental University, Nigata, Japan \\ ${ }^{2}$ Department of Physiology, School of Life Dentistry at Niigata, The Nippon Dental University, Nigata, Japan \\ ${ }^{3}$ School of Medical Technology, Gunma Paz College, Gunma, Japan \\ Email: aoy@ngt.ndu.ac.jp
}

Received 26 January 2015; accepted 29 April 2015; published 5 May 2015

Copyright (C) 2015 by authors and Scientific Research Publishing Inc.

This work is licensed under the Creative Commons Attribution International License (CC BY).

http://creativecommons.org/licenses/by/4.0/

(c) (i) Open Access

\begin{abstract}
The purpose of this study was to show the benefit of visualizing a tree-dimensional (3D) image of the tongue's muscle structure, which until now has been regarded as fully understood. Until now, no suitable 3D observation methods have been developed for soft tissue, such as the tongue, using histological magnification. For this purpose, this study used a micro-computed tomographic method (micro-CT) and image processing after the fixation, decalcification, and dehydration of a mouse tongue. Results: 3D rendered images of tongue muscles obtained by micro-CT showed every muscle and their relationships to each other. The superior longitudinal and the hyoglossus muscles of the tongue made up one group, while the inferior longitudinal and the styloglossus muscles of the tongue made up another. The boundary of the two muscles in each group was difficult to distinguish. On the other hand, what appear to be newly described muscles were identified. These results indicate that our micro-CT method is beneficial and that classical knowledge of tongue muscles derived from two-dimensional (2D) images does not fully describe the actual complexity of the tongue muscles. In our opinion, 3D rendered images mixed with raw structure can provide a more in-depth picture of the tongue from an integrated as well as an analytical perspective.
\end{abstract}

\section{Keywords}

Tongue Muscles, Micro-CT, Three-Dimensional Architecture, Longitudinal Muscle

\section{Introduction}

Anatomical and histological studies of the tongue began as early as the second century [1] and that work essen-

\footnotetext{
${ }^{*}$ Corresponding author.
}

How to cite this paper: Aoyagi, H., Iwasaki, S. and Asami, T. (2015) Three-Dimensional Architecture of the Tongue Muscles by Micro-CT with a Focus on the Longitudinal Muscle. Surgical Science, 6, 187-197. 
tially provided the definitive knowledge of the anatomy of the human tongue [2] [3]. The more recent morphological studies of human [4] and animal tongues [5]-[8] have had a greater focus on function. Examples include studies on tongue function in animals such as the chameleon and the toad [9]-[12]. Many physiological studies [13]-[15] have also focused on the function of the tongue in actions such as speaking, swallowing, and eating. Structural details of the tongue muscle, with a focus on tongue muscle innervation, have also been well studied [16]. The main reason for the sluggishness in morphological study of the tongue, despite active study of its physiology, is that no new observation methods have been developed that are suitable for studying the complex structure of the tongue.

One answer is 3D observation because a much greater amount of morphological information is available in 3D than in 2D images [17]-[19]. Today, 3D morphological studies of the tongue muscles are accomplished primarily using macro-anatomical [4] [20]-[22], histological [1] [6], computed tomography (CT) [23], magnetic resonance imaging (MRI) [13] [24] [25], and ultrasound methods [26]. However, most of these methods do not display histological resolution. On the other hand, Aoyagi showed the appropriateness of observations made using micro-CT [19], although he did not provide 3D images of individual tongue muscles. By contrast, reptilian tongues, such as that of the chameleon, have been successfully illustrated [5]. Rendered images like those shown in [27], in addition to illustrations, would provide a better depiction of the tongue structure. The aim of the present study was to achieve this goal using the mouse tongue muscle as a source of rendered images. For the first step, we obtained 3D rendered images in the focused longitudinal muscle (Lo) and its relational muscles such as the styloglossus (Sty) and hyoglossus (Hyo) muscles and discussed.

\section{Materials and Methods}

Animals and experimental procedures: Animal experiments were performed in accordance with the guidelines for animal experiments of Nippon Dental University's School of Life Dentistry at Niigata. Four 3-month-old mice (Slc: ddY, Hamamatsu, Japan) were used in this study. They were killed by an intraperitoneal overdose of sodium pentobarbital (200 mg/kg body weight). The head portions, with tongues, were fixed in $4 \%$ formaldehyde for 1 week at room temperature. The tongue was separated from the head and was decalcified in decalcifying solution A (Plank-Rychlo; Wako, Osaka, Japan). After alcohol dehydration, T-butyl alcohol substitution was performed. The tongue was then freeze dried in a freeze dryer (ES-2030; Hitachi, Hitachi, Japan).

Observations and image processing specimens were scanned using a micro-CT system (SMX-100CTSV, Shimadzu, Kyoto, Japan) as follows: X-ray source $22 \mathrm{kv}, 57 \mathrm{~mA}$, pixel size 512, 512, the source to object distance (SOD) $37.6 \mathrm{~mm}$, the source to image-receptor distance (SID) $179.7 \mathrm{~mm}$ (Figure 1(A), Figure 5), X-ray source 22 kv, $83 \mathrm{~mA}$, pixel size 512, 512, SOD $13.3 \mathrm{~mm}$, SID $216.3 \mathrm{~mm}$ (Figure 1(B), Figure 1(C), Figure 2, Figure 3), X-ray source 22 kv, $56 \mathrm{~mA}$, pixel size 512, 512, SOD $17.5 \mathrm{~mm}$, SID $179.8 \mathrm{~mm}$ (Figure 1(D), Figure 4). Micro-CT images were then reconstructed using 3D structural analysis software (MPR, Shimadzu, Kyoto, Japan and TRI/3D BON and FCS4D/VOLvie, Ratoc System Engineering, Tokyo, Japan).

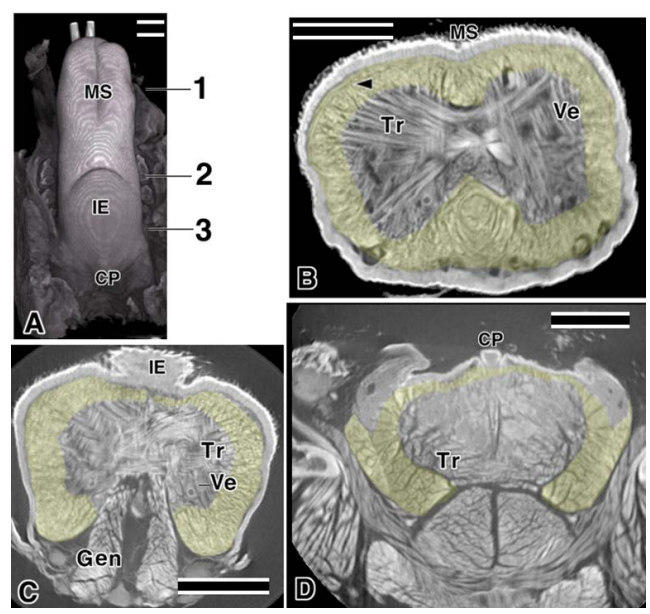

Figure 1. Mouse tongue image using micro-CT yellow; image processing areas. Bar; $10 \mu \mathrm{m}$. (A) Dorsal tongue; (B) Coronal cut face at 1; Arrowhead: mST; (C) Coronal cut face at 2; (D) Coronal cut face at 3. 


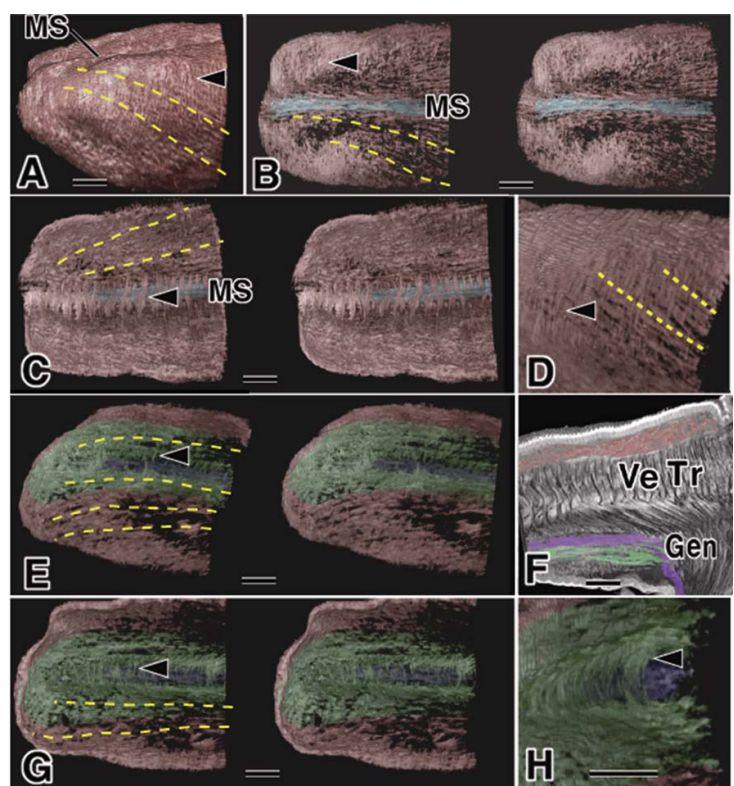

Figure 2. Apical part of the tongue using micro-CT. Red: sLo group, Green: iLo group, Yellow broken lines; the direction of muscle fascicles, Blue: mMS below MS, Purple: Gen. A pair of pictures: stereo-pair of images. Bar: $10 \mu \mathrm{m}$, (A) Apical part, Arrowhead: mST; (B) Dorsal part. Arrowhead: mST; (C) Inside view. Arrowhead: Jointed between of the sLo; (D) Strong transmitted image of (A). Arrowhead: mST; (E) Inferior part of (A). Arrowhead: Jointed between iLo; (F) Tomography sagittal cut with Gen. Arrowhead: same as (E), (G). Inside view. Arrowhead: Surrounding the Gen; $(\mathrm{H})$ Enlargement (G). Arrowhead: same as (G).

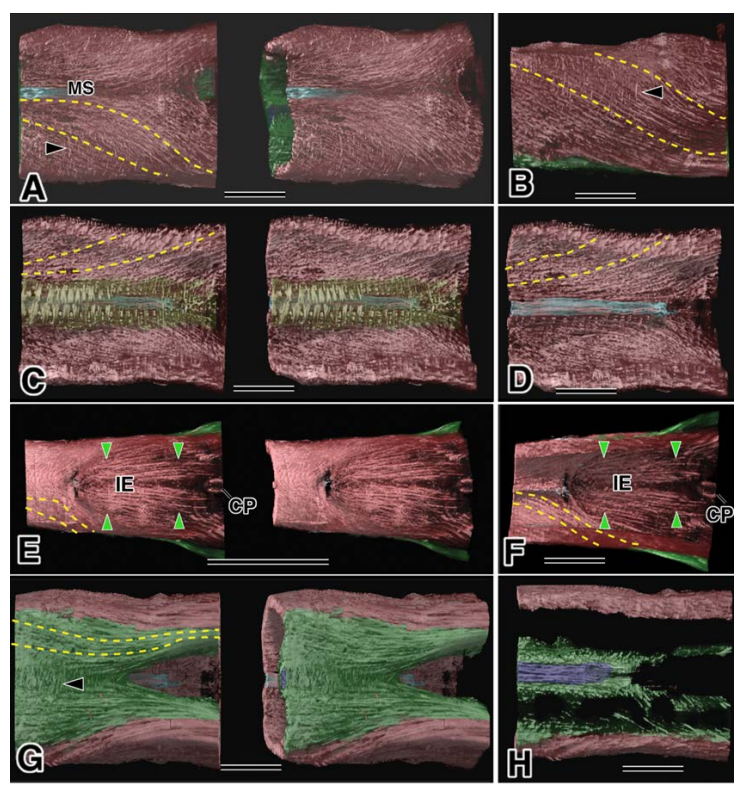

Figure 3. Middle part of the tongue using micro-CT. Red: sLo group, Green: iLo group, Yellow broken lines: the direction of muscle fascicles, Blue: mMS below the MS, Purple: Gen. Bar: $10 \mu \mathrm{m}$. (A) Middle dorsal part, with the mMS, mIE and iLo groups. Arrowhead: mST; (B) Lateral view with iLo group. Arrowhead: mST; (C) Inside view surrounding the mMS: in yellow; (D) After removal of the yellow part; (E) Middle dorsal part with iLo group. Green arrowheads: mIE near IE; (F) Inside view. Green arrowheads: same as (E), (G). bottom view. Arrowhead: jointed between the iLo group; (H) Horizontal cut through the Gen. 


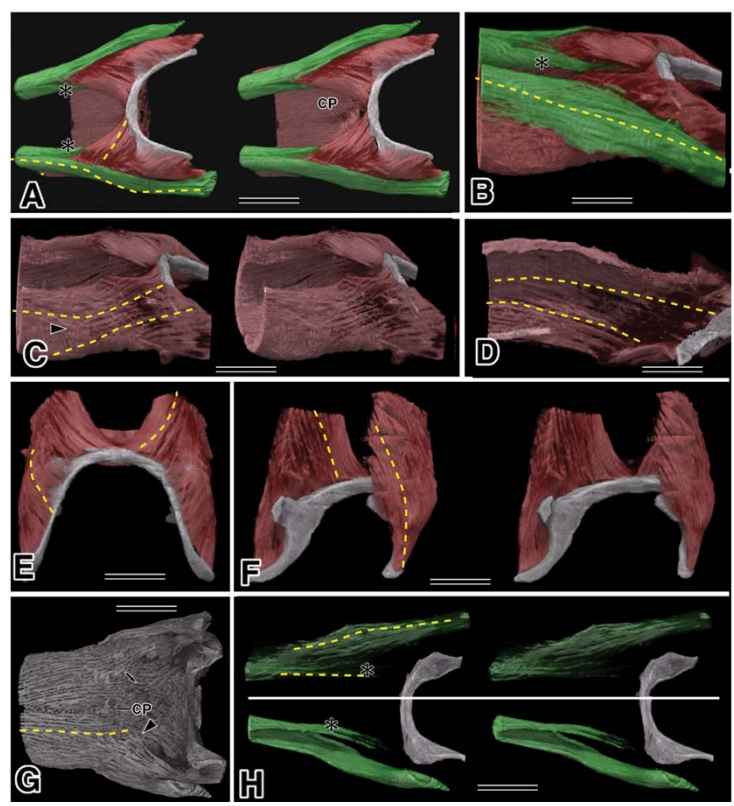

Figure 4. Root part of the tongue muscle by micro-CT. Red: sLo group, Green: iLo group, Yellow broken lines: the direction of muscle fascicles, White: HB, " mark: iLo. Bar: $10 \mu \mathrm{m}$. (A) Inferior root part with the CP and HB; (B) Lateral view; (C) After removal of the iLo group; (D) Inside view; (E) Posterior side view; (F) Oblique inside view; (G) Surface image, Arrowhead: Hyo terminated, Arrow: mIE terminated in the Tr; $(H)$ After removal of the sLo group, the upper: strong transmitted image; lower: weak transmitted image.

\section{Results}

\subsection{Extraction of the Image Objects}

The tongue muscles and their surrounding tissues were recorded using micro-CT and the Aoyagi method [19]. Two types of observation were performed: a low magnification for the entire tongue image (Figure 1(A), Figure 5) and a high magnification for the muscle fascicle images (Figures 1(B)-(D), Figures 2-4). In this study, we focused on the muscle fascicles that ran from anterior to posterior, such as the Lo, Sty, and Hyo. Those muscles were roughly selected in the tomography of the micro-CT and were highlighted in yellow (Figures 1(B)-(D)). $\operatorname{Tr}$ present in the region of interest were bypassed in the cases where the Tr ran into the Lo (Figure 1(B) arrowhead). In this research, all epithelium images were cropped by image processing and two types of depictions are presented in the Figs. The first type was a weak transmitted image: the figure near the surface in the 3D images was emphasized and most of the images were this type. The second type was a strong transmitted image: the figures in the deep areas in the 3D image were shown in addition to the surface ones. In several cases, the figures were shown as convergent stereo-pairs of reconstructed images.

\subsection{Observation of the Apical Part of the Tongue}

The tongue muscles on the coronal cut face were categorized into two types of muscles: a muscle around the tongue containing mainly the Lo and highlighted in yellow (Figure 1(B)) and a muscle in the center of the tongue consisting mainly of the Tr and Ve muscles. This method of separation was supported by the scanning electron microscope images [28]. A lateral view of the tongue showed that the muscles that surround the tongue (tentatively designated as the mST) were located on the outermost surface of the tongue (Figure 2(A) arrowhead), and obscured part of the muscle fascicles of the sLo (Figure 2(A), broken line). The mST might be connected with the $\operatorname{Tr}$ (Figure 1(B)-arrowhead), but a more detailed observation was not possible in this paper. The top view of the dorsal tongue showed the muscle fascicle highlighted in blue (tentatively identified as the mMS) clearly evident under the MS of the tongue (Figure 2(B)). Other muscle fascicles (Figure 3(B) broken lines) ran along the mMS. The muscle fascicle running in the same direction as Figure 2(B) and the mMSand that was 
surrounded by Tr or Vewasclearly evident in the inside view of the tongue (Figure 2(C)). The strong transmitted image in Figure 2(A) showed a clear view of thismuscle fascicle (Figure 2(D)). The information aboutthe running direction of the fascicles in the bottom view of the inferior part of the tongue (Figure 2(E)) and the structure in the coronal section (Figure 1(B)) led us to categorize another group of muscle fascicles, highlighted in green, as the iLo group (Figure 2(E)). We then used two temporary names: one was the sLo group that had the iLo and Hyo; the other was the iLo group that had the iLo and Sty. In the green area of Figure 2(E), we identified another clearly independent muscle fascicle, indicated in purple. This must be at least part of the Gen, judging from the sagittal cut bymicro-CT tomography ofthe Gen (Figure 2(F)). Some muscle fascicles that joined fascicles of the iLo group under the inferior part of the tongue and some muscle fascicles that surrounded the Gen were viewed around the Gen from the inside view of the tongue (Figure 2(G), Figure 2(H); arrowhead). The boundary between the sLo and iLo groups was not perfectly clear, but at least two types of muscle groups were evident.

\subsection{Observation of the Middle Part of the Tongue}

Observation of the dorsal part of tongue, including the end part of the MS and the IE [29] area, indicated that the sLo group of muscle fascicles runs from the anterior part to the posterior inferior part and some fascicles of thesLo group run close to the mMS and/or join with the mMS (Figure 3(A)). These relationships were easily recognized from the lateral view (Figure 3(B)) and by observation of aninside view of the tongue (Figure 3(C), (D)). Most of the mSTwasfound in the apical part of the tongue (Figure 2(A), Figure 2(D), Figure 3(A)). The end part of the mMS was not clear in this study (Figure 3(A), Figure 3(B)-arrowhead). Observation of the dorsal part near the IE indicated an arrangement of muscle fascicles suggestive of a new muscle group, where the muscle fascicle started from the edge of the IE and extended to the root part of the tongue atthe Tr. We called this muscle group tentatively the mIE. This mIE was also examined from an inside view of the tongue (Figure $3(\mathrm{~F})$ ). Observation of the inferior part of the tongue, as shown in Figure 3(A), indicated that most of the muscle fascicles of the iLo group ran symmetrically along the long axis of the tongue and were separated by the Gen, which isnot shown (Figure 3(G)). Each symmetrical muscle fascicle in the iLo group was joined by the small fascicles (Figure 3(G)-arrowhead). Part of the Gen that existed in the deeper part behind the iLo group and small fascicles was clearly shown in a horizontal cut through the Gen. The end part of the Gen disappeared because of its flexion toward the front(Figure 3(H)).

\subsection{Observation of the Root Part of the Tongue}

Observation of the root part of the tongue indicated that the iLo group and the sLo group were separated from each other (Figure 4(A)). The iLo group was clearly split into two parts: one was the so-called Sty (Figure 4(B)) and the other was probably the so-called iLo that ran into the root part of the tongue of the $\mathrm{Tr}$ and disappeared (Figure 4; *mark). The sLo group alone was shown by removing the iLo group with theimage analyzer and then the sLo group was viewed from several angles: lateral (Figure 4(C)), lateral inside view of the tongue (Figure 4(D)), posterior inferior (Figure 4(E)), and oblique posterior (Figure 4(F)). The muscle fascicles of the sLo group, which run from the hyoid bone (HB) roughly parallel to the long axes of the tongue and were designated Hyo in this area, were easily recognized, especially in the side view of the tongue and around the hyoid bone. The borderline between the sLo and Hyo in the sLo group was not clear, but part of muscle fascicles of the Hyo were observed by a non-transmitted image of micro-CT to terminate on the surface of the dorsal part of the tongue (Figure 4(G)). Some of the terminals of the mIE fascicles ran into the $\mathrm{Tr}$ and disappeared (Figure 4(G)arrow). The iLo group was alone and appearedas a strongly transmitted image in the upper half of Figure 4(H) and a weakly transmitted image in the lower half. This comparison readily revealed the muscle fascicles of the iLo group. One end portion of the iLo group was obviously the Sty and the other was probably the iLo, but the borderline of these in the iLo group was not clear in this figure (Figure 4(H)).

\subsection{Overall Observation of the Tongue}

Rendered images of the full tongue were obtained in the same manner as the partial tongue regionsand were modified using the results fromthe partial regions. The top view of the dorsal part of the tongue showed that the sLo group of muscles was viewed as containing the MS, mIE, CP, HB, and part of the iLo group but could not 
be shown at the resolution of muscle fascicles, so we added some broken lines in the figure to show the muscle fascicle directions (Figure 5(A)). The relationship between the sLo and iLo groups waswell recognized from the top view (Figure 5(A)), bottom view (Figure 5(B)) and/or lateral view (Figure 5(C)). We added help lines for each case, as in Figure 5(A). Separate images of the sLo and iLo groups are shown (Figure 5(D), Figure 5(E)) with guide lines, but the borderlines among the sLo, Hyo, mMS and/or mIE in the sLo group orthe iLo and Sty in the iLo group could not be depicted (Figure 5).

\section{Discussion}

Our aim in conducting this study was to show the benefit of 3D image visualization of the tongue muscle structure that has been regarded as fully understood. The first step to verify a benefit was to use micro-CT to study the mouse tongue. We obtained 3D rendered images associated with thesLo, iLo, Sty, Hyo, and/or part of the Gen, where muscle fascicles run in a direction essentially along the apex to the root of the tongue. We were unable to affirm a locational relationship among these muscles but we were able to separate the muscles into two groups. We have used a temporary classification of the sLo group instead of thesLo and Hyo and, similarly, of the iLo group instead of theiLo and Sty (Figure 5). We could not find this type of classification in the available literature or when using observation methods such as a total view of the tongue muscle. These discrepancies complicated the precise definition of the area corresponding to the sLo and iLo. In order to compare our results withthe existing knowledge, we divided these issues further, as follows: 1) An unclearly defined area between the sLo and iLo; 2) The root tongue area with an unclear relation between thesLo (or iLo) and Hyo and an unclear origin of the sLo; 3) The apical tongue area with an unclear relation among the iLo, Gen, and Sty; 4) A necessary adoption of new muscle names-mIE, mMS and/or mST - in Figure 1, Figure 2 and/or Figure 3. These problems indicated in issues 1) - 4) were strongly related to each other but are discussed separately.

1) Unclearly defined area between the sLo and iLo.

Most of the available literature involved examination of the mammalian sLo and iLo using coronal sections of 2D images of the anterior part of tongue, but the borderlines were unclear [21] [30]. McClung [21] insisted that deciding on the borderline between these two was difficult and that the separation marker for the two would be a vessel. The relations between the sLo and iLo were also indicated by each different colored position and the sLo and iLo were well separated at the apical region of the flattened tongue of a human fetus at age 32 weeks [31]. The problem of defining the area between the sLo and iLo arose both from species differences as well as from depiction differences, such as the use of 2D and 3D images. Takemoto et al. [32] [33], in their studies of human and chimpanzee tongues, showed 3D images of the sLo that were similar to our images of the anterior part of the tongue but they did not revealany clear relationship between the sLo and iLo at the posterior part of the tongue. In our case, the borderline of the two types of muscle (the sLo and iLo groups) was uncertain at the anterior part

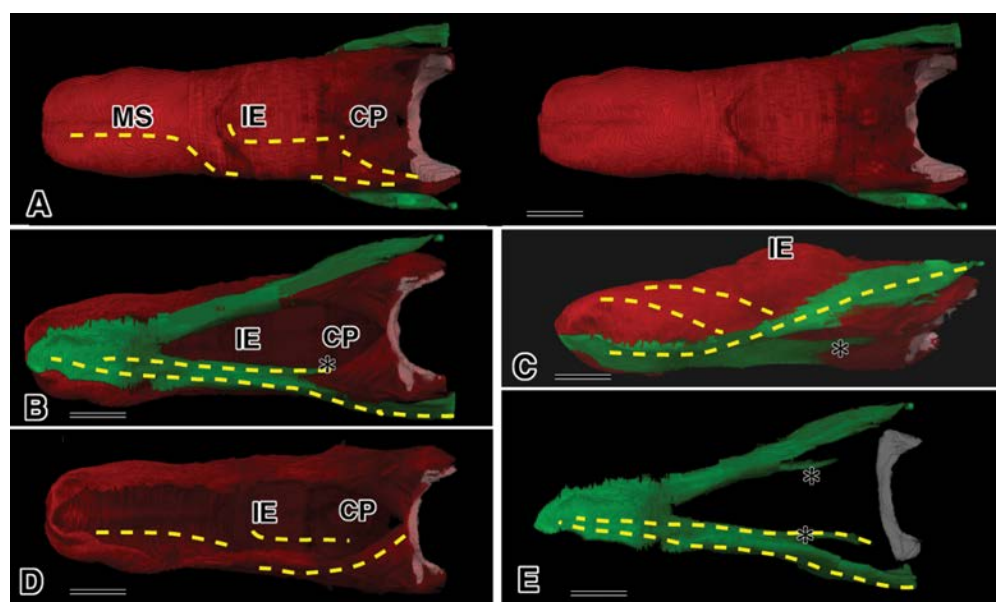

Figure 5. Total rendered images of the mouse tongue muscles. Red: sLo group, Green: iLo group, Yellow broken lines: the direction of muscle fascicles. *mark: iLo. Bar: $30 \mu \mathrm{m}$. (A) Dorsal part; (B) Inferior part; (C) Lateral part; (D) Separated iLo group; (E) Separated sLo group. 
of tongue because of the partial intermixture of fiber fascicles, but we could confirm the existence of two types of muscle fascicle at least in the area of the apical part of the tongue (Figure 2). McClung [21] showed that the Lo fibers run along the entire circumference of the tongue. In their depiction, they used the designation "Longitudinal muscle" instead of sLo and iLo. Selection of these words (sLo, iLo, and Lo), in our opinion, was a realistic choice but the terms must first be clearly defined.

2) Root tongue area with an unclear relation between the sLo [or iLo] and Hyo and an unclear origin of the sLo [or iLo].

The structural relations between the iLo and Hyo were well illustrated and indicatedthat the Hyo ran into the iLo (not the sLo) [2] [30] [34]-[36]. The 3D depiction of the iLo and Hyo showed that these overlapped each other. On the other hand, the Hyo terminated in the root part of the Tr and while the termination of the iLo in the area of the root tongue was unclear (in the case of humans [32] [37] of chimpanzees [27] [33]). In our case, the muscle fascicles continued from the whole surface of the hyoid bone to the apical part of the tongue for thesLo group but not forthe iLo group. The connection area between the sLo and Hyo was therefore unclear. For the iLo (Figure 4 and Figure 5; "mark), we thought that the ${ }^{*}$ mark in Figure 4 and Figure 5 partly indicated the socalled "iLo," based on a comparison of the results from a human case [30], but we could not find a similar published result for a mouse case. In relation to this finding, Sokoloff [38] also showed that the posterior intrinsic muscle fibers of the iLo and sLo could have attachments to the hyoid bone.

3) Apical part of the tongue with an unclear relation among the iLo, Gen, and Sty.

The Sty runs laterally into the inferior and anterior part of the human tongue from posterior part of the tongue [2] [32] and the muscles line up from the lateral to the central Sty, iLO, and Gen, in that order, on the inferior part of the coronal cut face [30] [31]. Miyawaki [35] added the Hyoto this area. The results of McClung [21] regarding the iLo position in the rat contrasted with our result that it was the Gen. The ventrally running Sty and iLo interdigitate with each other [21]. The total view shown in Figure 5 depicts the Sty as a part of theiLo group, running from the lateral side into the apical part, but the boundary between iLo and Sty was not distinguished.

4) Necessary adoption of new muscle names in Figure 1, Figure 2 and Figure 3.

Figure 3(E) and Figure 3(F) show the muscle arrangement near the IE structure as the sLo group, but no evidence of this muscle was previously published, so we used the temporary name "mIE." Many earlier researchers were probably not concerned about this structure because it was not apparent in 2D observations, and they considered the mIE to be the sLo. A developmental study of tongue muscle would be needed to clarify this issue.

The arrowheads in Figure 1(B), Figure 2(A) and Figure 2(B), and Figure 2(D), Figure 3(A) and Figure $3(B)$, and Figure 4(C) indicate muscle fibers surrounding the surface of the tongue with observed connections to the Tr. No special name was given previously but a few observations were published [38]. These muscle fascicles appeared to have the function of collecting up the sLo, so we used the temporary name "mST." Additional searches are needed to confirm this function. The muscle highlighted in blue in Figure 2(B) and Figure 2(C) and Figure 3(A), Figure 3(C), and Figure 3(D) attracted our attention because it was surrounded by the Tr and some researchers have designated it as the sLo [21]. This muscle was intermixed with the sLo to some extent, but it is likely to have a special function, so we used the temporary name "mMS".

These problems 1) - 4) can only be resolved by additional research, such as obtaining histological proof, but we would like to emphasize that $3 \mathrm{D}$ images of the tongue muscle are more helpful than $2 \mathrm{D}$ from an integrated perspective as well as an analytical one. Our consideration of these problems from another point of view also led to the conclusion that the fiber architecture [38] is important: the muscular hydrostat model is one example [21] [39]. On the other hand, recent advances in the field of cell engineering, by Okuda [40], for example, now allow modeling of cell proliferation for simulating three-dimensional tissue morphogenesis based on a reversible network reconnection framework. If we were to apply this type of model to muscle structure, we could consider the muscle structure of tongue as a new muscle fascicle architecture. In this case, our model of the tongue muscle would be effective. Taken together, our results (Figure 5) suggest a rational structure for the fiber architecture of the tongue.

\section{Observation Methods for Tongue Muscles}

The study of the complex structure of the tongue muscles relies on the following three points: 1) The method of acquisition of the tongue muscle; 2) The methods of image analysis; and 3) The display methods used for the parsed images in the paper. 
1) Method of acquisition of the tongue muscle

The use of micro-CT, instead of MRI [13] [24] [25], ultrasound [26] etc., is presently the best choice for fine observation of the complex muscle structure of the tongue [19]. Considering the computer capability in the average laboratory, analyzing a total sample may prove difficult, as working with high-resolution images at same timerequires a large computing capacity. We therefore cropped the images into several portions (Figures 2-4) and then integrated them (Figure 5). In some areas, such as indicated in Figure 2(A) by the arrowhead, a higher-resolution image [19] will be needed, but this will be addressed in our next study.

2) Method of image analysis

The tongue is essentially a mass of complex muscles, so rendering the muscle units is difficult from the density information of micro-CT only. The structural information of the muscle fascicles is then a useful addition to the density information. Miyawaki [35] focused on the muscle fascicle direction but he was unable to use 3D images. Today, the use of MRI allows the muscle fascicle direction to be obtained easily and the muscles rendered [34], but the histological resolution is not sufficient. If we could trace one muscle fascicle from start to finish, then evencomplex muscles would be easily separated; however, muscle fascicles unite and separate repeatedly. Therefore, at present, we have only focused on muscle delectation but not onindividualunit fascicles of the muscle. The possibility exists, therefore, that a small number of muscle fascicles in the mass of the sLo group or the iLo group could cross each other, and these cross relations were not checked during image processing. This challenge of the complexity of muscle fascicles will be the focus of a subsequent study.

3) Method of display of the parsed image in paper

All structure information is replaced by numerical information in case of micro-CT, but there is not enough room to show the 3D images in the paper [17]-[19]. In general, our ability for structural recognition precludes viewing all structures from the surface throughout deeper areas at same time, whereas imaging the curved surface of the structure is possible in three-dimensional space. Therefore, as a matter of course, we can only select a few images from the extensive micro-CT data to demonstrateour concept. The most important idea is thatmicro-CT data has the capability of revealing much morestructural information. In this study, as a first step, we focused mainly on the sLo, iLo, Hyo, and/or Sty and we will generate muscle images of the entire tongue as the next step.

\section{Conclusion}

Micro-CT can generate 3D rendered images of tongue muscles that allow imaging of every muscle to show their relationships to each other. The superior longitudinal and the hyoglossus muscles of the tongue made up one group, while the inferior longitudinal and the styloglossus muscles of the tongue made up another. These results indicate that our method is beneficial and that classical knowledge of tongue muscles derived from 2D images does not fully describe the actual complexity of the tongue musculature.

\section{Acknowledgements}

We are grateful to Prof. M. Tsuchimochi of the Department of Oral and Maxillofacial Radiology of the Nippon Dental University School of Life Dentistry at Niigata for his encouragement and constant support. We also wish to thank Prof. A. Ezura of General Dentistry at the Nippon Dental University School of Life Dentistry at Niigata for his help and suggestions. This research was supported by Research Promotion Grants [No. NDUF-14-13] from the Nippon Dental University.

\section{References}

[1] Barnwell, E.M. (1976) Human Lingual Musculature: an Historical Review. International Journal of Oral Myology, 2, 31-41.

[2] Clemente, C. (1985) Gray’s Anatomy of the Human Body. 30th Edition, Lea \& Fibiger, Philadelphia.

[3] Schaffer, J. (1951) Collective Review, Clinical Pathology of the Tongue. Oral Surgery, Oral Medicine, Oral Pathology, 4, 1287-1316. http://dx.doi.org/10.1016/0030-4220(51)90088-6

[4] Ogata, S., Mine, K., Tamatsu, Y. and Himada, K. (2002) Morphological Study of the Human Chondroglossus Muscle in Japanese. Annals of Anatomy, 184, 493-499. http://dx.doi.org/10.1016/S0940-9602(02)80087-5

[5] Diogo, R. (2009) The Head and Neck Muscles of the Philippine Colugo (Dermoptera: Cynocephalus valans), with a 
Comparison to Treeshrews, Primates, and Other Mammals. Journal of morphology, 270, 14-51. http://dx.doi.org/10.1002/jmor.10666

[6] Smith, J.C., Goldberg, S.J. and Shall, M.S. (2006) Myosin Heavy Chain and Fiber Diameter of Extrinsic Tongue Muscles in Rhesus Monkey. Archives of Oral Biology, 51, 520-525. http://dx.doi.org/10.1016/j.archoralbio.2005.10.006

[7] Smith, K.K. (1988) Form and Function of the Tongue in Agamid Lizards with Comments on Its Phylogenic Significance. Journal of Morphology, 196, 157-171. http://dx.doi.org/10.1002/jmor.1051960205

[8] Schwenk, K. (1994) Why Snakes Have Forked Tongues. Science, 263, 1573-1577. http://dx.doi.org/10.1126/science.263.5153.1573

[9] Gans, C. and Gorniak, G.C. (1982) Functional Morphology of Lingual Protrusion in Marine Toads [Bufo marinus]. American Journal of Anatomy, 163, 195-222. http://dx.doi.org/10.1002/aja.1001630302

[10] Herrel, A., Meyers, J.J., Nishikawa, K.C. and Vree, F.D. (1986) Morphology and Histochemistry of the Hyolingual Apparatus in Chameleons. Journal of Morphology, 249, 154-170. http://dx.doi.org/10.1002/jmor.1047

[11] Smith, K.K. (1986) Morphology and Function of the Tongue and Hyoid Apparatus in Varanus (Varanidae, Lacertilia). Journal of Morphology, 187, 261-287. http://dx.doi.org/10.1002/jmor.1051870302

[12] Lombard, R.E. and Wake, D. (1977) Tongue Evolution in the Lungless Salamanders, Family Plethodontidae. II. Function and Evolutionary Diversity. Journal of Morphology, 153, 39-80. http://dx.doi.org/10.1002/jmor.1051530104

[13] Sosnovik, D.E., Wang, R., Dai, G., Wang, T., Aikawa, E., Novikov, M., Rosenzweig, A., Gilbert, R.J. and Wedeen, V.J. (2009) Diffusion Spectrum MRI Tractography Reveals the Presence of a Complex Network of Residual Myofibers in Infracted Myocardium. Circulation: Cardiovascular Imaging, 2, 206-212. http://dx.doi.org/10.1161/CIRCIMAGING.108.815050

[14] Kayalioglu, M., Shcherbaty, V., Seifi, A. and Liu, Z.J. (2007) Roles of Intrinsic and Extrinsic Tongue Muscles in Feeding: Electromyographic Study in Pigs. Archives of Oral Biology, 52, 786-796. http://dx.doi.org/10.1016/j.archoralbio.2007.01.004

[15] Napadow, V.J., Chen, Q., Wedeen, V.J. and Gilbert, R.J. (1999) Intramural Mechanics of the Human Tongue in Association with Physiological Deformations. Journal of Biomechanics, 32, 1-12. http://dx.doi.org/10.1016/S0021-9290(98)00109-2

[16] Mu, L. and Sanders, I. (1999) Neuromuscular Organization of the Canine Tongue. Anatomical Record, 256, $412-424$. http://dx.doi.org/10.1002/(SICI)1097-0185(19991201)256:4<412::AID-AR8>3.0.CO;2-5

[17] Aoyagi, H., Tsuchikawa, K. and Iwasaki, S. (2010) Three-Dimensional Observation of the Mouse Embryo by MicroComputed Tomography: Composition of the Trigeminal Ganglion. Odontology, 98, 26-30. http://dx.doi.org/10.1007/s10266-009-0112-9

[18] Aoyagi, H., Tsuchikawa, K. and Iwasaki, S. (2012) Three-Dimensional Observation of the Mouse Embryo by MicroComputed Tomography: Meckel’s Cartilage, Otocyst, and/or Muscle of Tongue. Odontology, 100, 134-143.

[19] Aoyagi, H., Iwasaki, S. and Nakamura, K. (2015) Three-Dimensional Observation of Mouse Tongue Muscles Using Micro-Computed Tomography. Odontology, 103, 1-8.

[20] McClung, J.R. and Goldberg, S.J. (2002) Organization of the Hypoglossal Motoneurons That Innervate the Horizontal and Oblique Components of the Genioglossus Muscle in the Rat. Brain Research, 950, 321-324. http://dx.doi.org/10.1016/S0006-8993(02)03240-7

[21] McClung, J.R. and Goldberg, S.J. (2000) Functional Anatomy of the Hypoglossal Innervated Muscles of the Rat Tongue: A Model for Elongation and Protrusion of the Mammalian Tongue. Anatomical Record, 260, 378-386. http://dx.doi.org/10.1002/1097-0185(20001201)260:4<378::AID-AR70>3.0.CO;2-A

[22] Saito, H. and Itoh, I. (2007) The Three-Dimensional Architecture of the Human Styloglossus Especially Its Posterior Muscle Bundles. Annals of Anatomy, 189, 261-267. http://dx.doi.org/10.1016/j.aanat.2006.10.002

[23] Stutley, J., Cooke, J. and Parsons, C. (1989) Normal CT Anatomy of the Tongue, Floor of Mouth and Oropharynx. Clinical Radiology, 40, 248-253. http://dx.doi.org/10.1016/S0009-9260(89)80184-9

[24] Laine, F.J. and Smeker, W.R.K. (1995) Oral Cavity: Anatomy and Pathology. Seminars in Ultrasound, CT and MRI, 16, 527-545. http://dx.doi.org/10.1016/S0887-2171(06)80024-7

[25] Boom, H.P.W., van Spronsen, P.H., van Ginkel, F.C., van Schijndel, R.A., Castelijns, J.A. and Tuinzing, D.B. (2008) A Comparison of Human Jaw Muscle Cross-Sectional Area and Volume in Long- and Short-Face Subjects, Using MRI. Archives of Oral Biology, 53, 273-281. http://dx.doi.org/10.1016/j.archoralbio.2007.08.013

[26] Volk, J., Kadivec, M., Music, M.M. and Ovsenik, M. (2010) Three-Dimensional Ultrasound Diagnostics of Tongue Posture in Children with Unilateral Posterior Crossbite. American Journal of Orthodontics and Dentofacial Orthopedics, 138, 608-612. http://dx.doi.org/10.1016/j.ajodo.2008.12.028

[27] Lautenschlager, S., Bright, J.A. and Rayfield, E.J. (2014) Digital Dissection-Using Contrast-Enhanced Computed 
Tomography Scanning to Elucidate Hard- and Soft-Tissue Anatomy in the Common Buzzard Buteo buteo. Journal of Anatomy, 224, 412-431. http://dx.doi.org/10.1111/joa.12153

[28] Saito, H. and Itoh, I. (2003) Three-Dimensional Architecture of the Intrinsic Tongue Muscles, Particularly the Longitudinal Muscle, by the Chemical-Maceration Method. Anatomical Science International, 78, 168-176. http://dx.doi.org/10.1046/j.0022-7722.2003.00052.x

[29] Iwasaki, S., Yosizawa, H. and Kawahara, I. (1997) Study by Scanning Electron Microscopy of the Morphogenesis of Three Types Lingual Papilla in the Rat. Anatomical Record, 247, 528-541. http://dx.doi.org/10.1002/(SICI)1097-0185(199704)247:4<528::AID-AR12>3.0.CO;2-R

[30] Abd-el-Malek, S. (1939) Observations on the Morphology of the Human Tongue. Journal of Anatomy, 73, $201-210$.

[31] Toure, G. and Vacher, C. (2006) Anatomic Study of Tongue Architecture Based on Fetal Histological Section. Surgical and Radiologic Anatomy, 28, 547-552. http://dx.doi.org/10.1007/s00276-006-0144-6

[32] Takemoto, H. (2001) Morphological Analyses of the Human Tongue Musculature for Three-Dimensional Modeling. Journal of Speech Language and Hearing Research, 44, 1-13. http://dx.doi.org/10.1044/1092-4388(2001/009)

[33] Takemoto, H. (2008) Morphological Analyses and 3D Modeling of the Tongue Musculature of the Chimpanzee (Pan troglodytes). American Journal of Primatology, 70, 966-975. http://dx.doi.org/10.1002/ajp.20589

[34] Gaige, T.A., Benner, T., Wang, R., Wedeen, Van J. and Gilbert, R.J. (2007) Three Dimensional Myoarchitecture of the Human Tongue Determined in Vivo by Diffusion Tensor Imaging with Tractography. Journal of Magnetic Resonance Imaging, 26, 654-661. http://dx.doi.org/10.1002/jmri.21022

[35] Miyawaki, K. (1973) A Study on the Musculature of the Human Tongue. Annual Bulletin (Research Institute of Logopedics and Phoniatrics), 8, 23-49.

[36] Hellstrand, E. (1980) Morphological and Histochemical Properties of Tongue Muscles in Cat. Acta Physiologica Scandinavica, 110, 187-198. http://dx.doi.org/10.1111/j.1748-1716.1980.tb06650.x

[37] Iskander, A. and Sandes, I. (2003) Morphological Comparison between Neonatal and Adult Human Tongue. Annals of Otology, Rhinology \& Laryngology, 112, 768-776. http://dx.doi.org/10.1177/000348940311200905

[38] Sokoloff, A. and Burkholder, S.A. (2013) Chapter 12. Tongue Structure and Function. In: McLoon, L.K. and Andrade, F.H., Eds., Craniofacial Muscles, Springer, New York, 207-227.

[39] Kier, W.M. and Smith, K.K. (1985). Tongues, Tentacles and Trunks: The Biomechanics of Movement in MuscularHydrostats. Zoological Journal of the Linnean Society, 83, 307-324. http://dx.doi.org/10.1111/j.1096-3642.1985.tb01178.x

[40] Okuda, S., Inoue, Y., Eiraku, M., Sasai, Y. and Adachi, T. (2013) Modeling Cell Proliferation for Simulating Three-Dimensional Tissue, Morphogenesis Based on a Reversible Network Reconnection Framework. Biomechanics and Modeling in Mechanobiology, 12, 987-996. http://dx.doi.org/10.1007/s10237-012-0458-8 


\section{Abbreviations}

Lo: Longitudinal muscle of the tongue

sLo: Superior longitudinal muscle of the tongue

iLo: Inferior longitudinal muscle of the tongue

Tr: Transverse muscle of tongue

Ve: Vertical muscle of the tongue

Sty: Styloglossus muscle of the tongue

Hyo: Hyoglossus muscle of the tongue

Gen: Genioglossus muscle of the tongue

MS: Median sulcus of the tongue

IE: Intermolar eminence

HB: Hyoid bone

CP: Circumvallate papilla

\section{Abbreviations of Temporary Names in This Paper}

sLo Group: sLo and Hyo

iLo Group: iLo and Sty

mIE: Muscle found under the IE

mMS: Muscle fascicle highlighted in blue under the MS

mST: muscles that surround the tongue 